\title{
FORMULATION OF A MIXED INTEGER LINEAR PROGRAMMING MODEL IN PLANNING THE EXPANSION OF A TRANSMISSION NETWORK
}

\section{POVEDA ROBERTO ${ }^{1}$, RIVAS TRUJILLO, EDWIN ${ }^{2}$ \& RAMÍREZ VANEGAS CARLOS ${ }^{3}$}

${ }^{I}$ Facultad de Ingeniería Universidad Distrital Francisco José de Caldas, Bogotá D.C

${ }^{2}$ Facultad de Ingeniería Universidad Distrital Francisco José de Caldas, Bogotá D.C, 11021, Colombia; GCEM Research Group

${ }^{3}$ Departmento de Matemáticas, Universidad Tecnológica de Pereira, Pereira, Colombia

\section{ABSTRACT}

In this article the formulation of a mixed integer linear programming model is developed for the planning of the expansion of the network, the Garver 6 node test system is taken as a case study where the feasibility of installing lines and / or BESS is evaluated. (Battery Energy Storage System) to supply the power to grid loads, while maximizing net social welfare.

KEYWORDS: BESS, Expansion of the Network, Power Flows Transmission Network

Received: Sep 24, 2020; Accepted: Oct 14, 2020; Published: Nov 09, 2020; Paper Id.: IJMPERDOCT202033

\section{INTRODUCTION}

Considering the challenges that power systems face to satisfy current and future demands, the formulation of a mixed integer linear programming model is presented in the planning of the expansion of transmission networks.

A formulation of a mixed integer mathematical linear programming model is developed that allows evaluating the economic and technical feasibility of incorporating energy storage systems, as well as evaluating its parameters in relation to the conditions of the network and its possible expansions [1-4].

The AC power flow is solved through non-linear programming methods (Newton Raphson) and represents the best approximation to the real conditions of the system, since the power flow developed by the DC model omits the losses of the system. The model developed in GAMS represents an intermediate term between AC and DC power flows by taking into account most of the considerations of the DC model, but considering the effect of losses on the flows through the lines.

\section{FORMULATION OF THE WHOLE MIXED PROGRAMMING MODEL}

Considering the market structure, a linear programming model is formulated to maximize the net social welfare in the planning problem of the expansion of a network through lines and / or batteries [1]. In addition to the linear programming model, this section presents the relationship between the equations presented and those introduced in the mixed integer programming model (MIP) developed in GAMS.

In (1), the objective function of the model that seeks to maximize net social welfare is established, expressed as the aggregate utility function of demand minus the aggregate utility function of supply plus the aggregate utility function of batteries that act as consumers, minus the aggregate utility function of batteries 
operating as generators, minus the cost of investment in new lines and batteries. To make investment and operating costs comparable $\sigma$ is equal to $1 / 8760$, where 8760 are the hours of the year.

$$
\begin{aligned}
& \sum_{\forall c \in \Omega_{c}} W c\left(\sum_{\forall d \in \Omega_{d}} \lambda_{D d}{ }^{c} p_{D d}{ }^{c}-\sum_{\forall g \in \Omega_{G}} \lambda_{G g}{ }^{c} p_{G g}\right) \\
&+\sum_{\forall\left(B_{m}\right) \in \Omega_{B}} W c\left(\sum_{\forall B m \in \Omega_{B}} \lambda_{B_{m}}{ }^{+c} p_{B_{m}}{ }^{+c}-\sum_{\forall B m \in \Omega_{B}} \lambda_{B_{m}}{ }^{-c} p_{B_{m}}{ }^{-c}\right) \\
&-\left(\tau_{\tau^{2} \varepsilon_{L} \sigma} \sum_{\forall(s r k) \in \Omega_{L^{+}}} K_{s r k} w_{s r k}\right) \\
&-\left(\sum_{\forall(B m) \in \Omega_{B^{+}}} T_{B m} y_{B m} D_{B} S_{B a}{ }^{m a x}\right)
\end{aligned}
$$

Where

$d$ is the i-th demand block.

$g$ is the i-th generation block.

$B_{m}$ is the i-th battery.

c Scenario

$\lambda_{D d}{ }^{c}$ Demand price of the i-th demand block in scenario c.

$\lambda_{G g}{ }^{c}$ Offer price of the i-th generation block in scenario c.

$\lambda_{B_{m}}{ }^{+c}$ Price when the i-th battery is charging.

$\lambda_{B_{m}}{ }^{-c}$ Price when the i-th battery is discharging.

$p_{B_{m}}{ }^{+c}$ Power flow in battery when charging.

$p_{B_{m}}{ }^{-c}$ Power flow into the battery when it is discharging.

$\tau_{L}$ Annual amortization rate of the new lines.

$\varepsilon_{L}$ Scaling factor for new lines.

$\varepsilon_{L}$ Scaling factor for new batteries.

$\sigma$ Factor to make investment and operating costs comparable.

$\Omega_{d}$ Set of all loads.

$\Omega_{G}$ Set of all generators.

$\Omega_{B}$ Set of all batteries.

$\Omega_{c}$ Set of all scenarios. 
$\Omega_{B}$ Set of all batteries.

$\Omega_{L}+$ Set of new lines.

$\Omega_{B}+$ Set of new batteries.

$s, r, k$ Reference the line $\mathrm{k}$ located between the nodes $s, r$.

$K_{s r k}$ Cost of the line

$w_{s r k}$ Binary variable that specifies if the line is constructed.

$y_{B m}$ Binary variable that indicates if the battery is working.

$\tau_{B}$ Annual amortization rate of new batteries.

$T_{B m}$ Binary variable that specifies the total cost of the new battery.

$W c$ Weight for each of the scenarios. It is specified that each of the 6 scenarios has the same weight, therefore $W c=1 / 6$.

In Figure 3, the aggregate utility function for the $\operatorname{SUM}((S, G)$ generation represents a double summation of all the elements that it has concatenated, in order of relevance, first it adds the elements in the set of generators for a given scenario, and then add the results of all the scenarios. The operations that are concatenated within the summations correspond to the weight of each scenario times 876000 (24 hours a day, 365 days a year, by the base of 100 to pass the values in pu to $M W$ ) by the power in each of the generators according to the scenario. The utility function for the power demand in the loads and for the power exchange between BESS and the grid depending on whether they are being loaded or unloaded, have the same structure as the generation utility function A planning horizon of one year has been considered.

Investment costs are also included in Figure 1. For lines, the amortization rate establishes the cost per year if a recovery of the total cost of the line is considered over a period of 25 years; While the binary variable wsrk is a variable associated with each corridor and the maximum number of lines per corridor, the logic for wsrk defines that the investment costs for lines do not take into account the lines already built before the expansion process. For investment costs in BESS, the amortization rate establishes the cost per year if a recovery of the total cost of the line is considered in a period of 10 years, the total costs of the battery are defined based on the maximum energy that can store, considering a degradation factor, the binary variable and defines the batteries that must be installed per node in the expansion process.

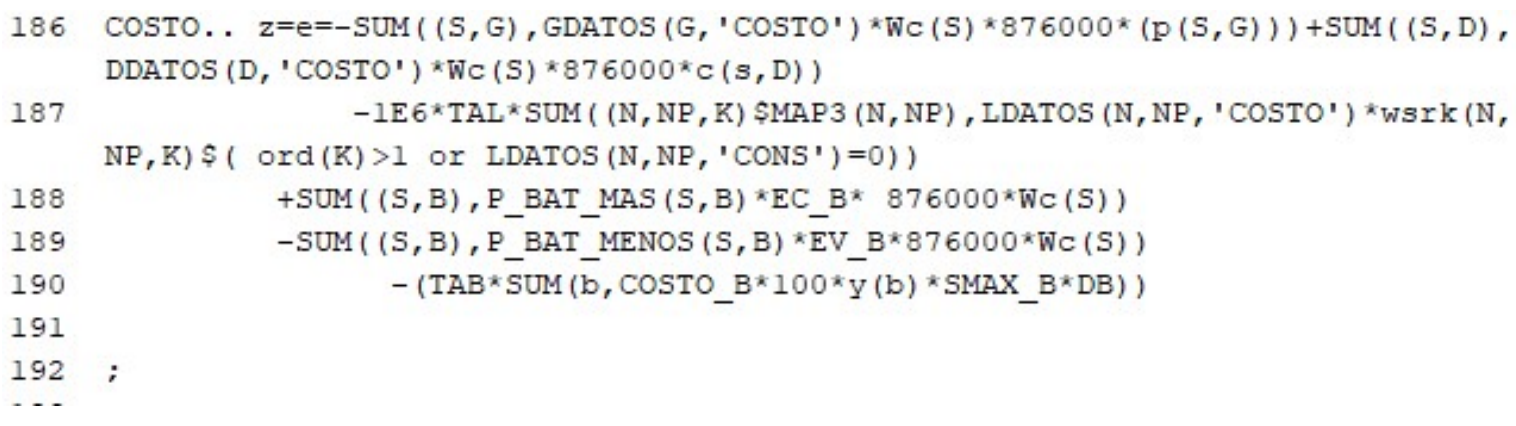

190

191

192 ;

-..

\section{Figure 1}

The restriction in (2) considers the power balance in each of the nodes of the system, taking into account that the sum of the power injected and delivered in the node must be equal to zero, considering that the losses in the lines are assumed equally by the nodes $s$ y $r$. 
Restrictions

$\sum_{\forall g \in \psi^{s}{ }_{G}} p G_{g}-\sum_{\forall(r . k) \in \psi^{s}{ }_{L}} f_{\text {srk }}+\frac{1}{2} q_{s r k}-\sum_{\forall g \in \psi^{s}{ }_{B}} p B_{m}=\sum_{\forall d \in \psi^{s}{ }_{D}} p D_{d}$

In Figure 2, the summation of the flows, generations and power demands is performed as long as they are connected to the node. The $\$$ sign is used as a conditional to select the elements that are associated with the node.

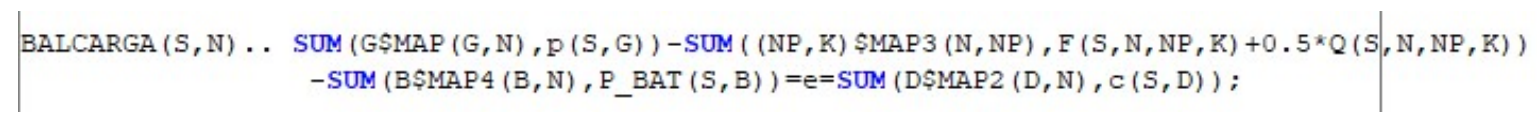

\section{Figure 2}

Where:

$\psi_{G}^{S}$ Set of all generators at node s

$\psi_{G}^{s}$ Set of all batteries in node s

$\psi_{L}^{s}$ Set of all lines that connect to node s

$\psi_{D}^{s}$ Set of demand blocks in bar s.

$r$ Node where the line starts.

$k$ Node where the line ends.

$f_{\text {srk }}$ Power flow through the line without considering losses.

$q_{\text {srk }}$ Power losses on the line.

In Figure 3 and (3) variables and relationships are introduced for linearization of the equations that describe the energy stored by a battery at an instant of time $t$. The notation $=E=$ is equivalent to equal.

$p B_{m}={p_{B_{m}}}^{+}-{p_{B_{m}}}^{-}$

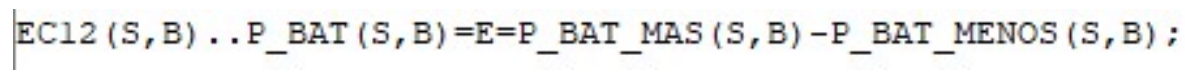

Figure 3

Where:

${p_{B m}}^{+}$Power flow when battery is charging.

$p_{B_{m}}{ }^{-}$Power flow when the battery is discharging.

In Figure 4, in addition to conditions (4) and (5), additional conditions are defined that prevent the model from assigning a power flow to batteries that are not built.

$p_{\text {srk }}^{\min } y p_{\text {srk }}^{\text {max }}$ are the thermal capacity limits of line $\mathrm{k}$ between nodes $\mathrm{s}$ and $\mathrm{r}$

In (4) if the battery is discharging $\left(y 1_{B_{m}}=1\right),{p_{B_{m}}}^{+}$is equal to zero, if $y 1_{B_{m}}=0$ the maximum power flow It is 
only limited by $p_{B_{m}}{ }^{\max }$, with the same approach, in (5) we set ${p_{B_{m}}}^{-}$it is limited by ${p_{B_{m}}}^{\max }$. Where $p_{B m}$ max is the absolute value of the maximum flow, either from or to the battery.

$0 \leq p_{B_{m}}{ }^{+} \leq\left(1-y 1_{B_{m}}\right) p_{B_{m}} \max$

$0 \leq p_{B_{m}}{ }^{-} \leq y 1_{B_{m}} p_{B_{m}}^{\max }$

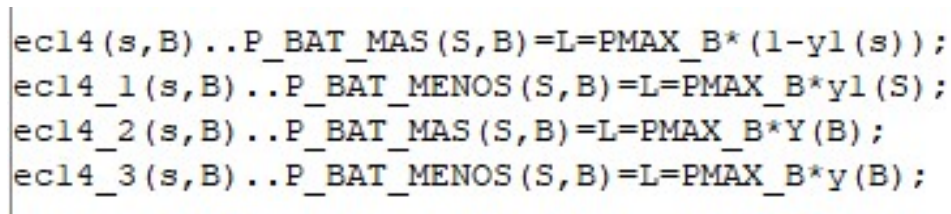

Figure 4

Equation (6) and (7) establish the restrictions for the power transmitted through the lines. In (7) it is taken into account that, if the line is constructed, the relationship between the power flow without losses, the susceptance of the lines and the angle of the bars, if the line is not constructed, the value of $\delta$ is given freedom $\delta^{s} y \delta^{r}$ to assume values given by other constraints. With a similar methodology, the restrictions are established to relate the losses with a value equal to 0 if line (9) is not built, or with (8) if the line is built.

In Figure 5 and Figure $6=L=$ is equivalent to the sign less than or equal to, while $=G=$ is equivalent to the sign greater than or equal to. In Figure 7 the summation is performed for each of the partitions that constitute the linearization process.

$p_{\text {srk }}^{\min } w_{\text {srk }} \leq f_{\text {srk }} \geq p_{\text {srk }}^{\max } w_{\text {srk }}$

$-(1-w s r k) M \leq \frac{f_{s r k}}{b_{s r k}}+\delta^{s}-\delta^{r} \leq(1-w s r k) M$

$0 \leq-\frac{q_{s r k}}{g_{s r k}}+\sum_{l=1}^{L} \delta_{s r}(l) \alpha_{s r}(l) \leq(1-w s r k) M$

$p_{\text {srk }}{ }^{\min } w_{\text {srk }} \leq q_{\text {srk }} \geq p_{\text {srk }}^{\max } w_{\text {srk }}$

Where:

$M$ is a constant 


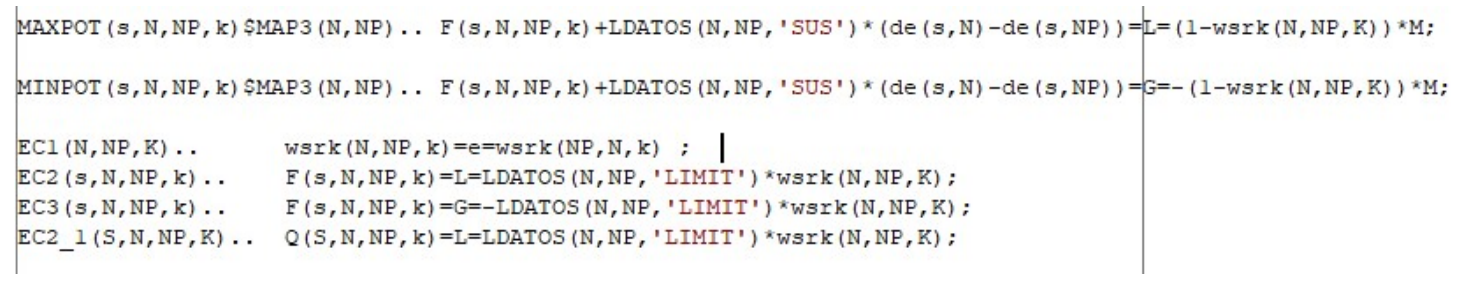

\section{Figure 5}

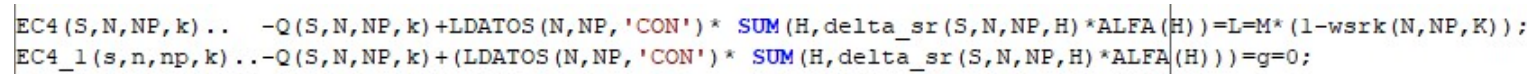

\section{Figure 6}

The total power that by thermal capacity can be transmitted through the line must be greater than the flow without losses that leaves node $\mathrm{s}$ through line $\mathrm{k}$ and the losses of line $\mathrm{k}$ assumed by the node, it is also taken into account that there are two possible directions for the flow exiting node s.

In Figure 7 the restrictions for the energy stored in the BESS are defined. Two conditions are generated for the relationship of stored energy between scenarios, the first one selects the scenarios smaller than 6 with the instruction to assign to the next scenario the energy that the battery already has stored in that scenario, plus the energy that it exchanges with the grid; while in the second condition when the scenario is equal to 6 , the energy that it has stored plus the exchange of energy with the network is assigned to the energy that the battery stores in scenario 1.

Comparing Figure 7 with their respective equations, the 0 has been replaced by a condition at minus infinity, so that the equations are applicable only if it meets the loading or unloading condition in the scenario.

$0<p D_{d}<p D_{d}^{\max }$

$0<p G_{g}<p G_{g}^{\max }$

$0 \leq S_{B m} \leq S_{B m} \max$

$0 \leq S_{B m}(t+1)-S_{B m}(t)-\Delta t{p_{B_{m}}}^{+}(t) \leq y 1_{B_{m}} M$

$0 \leq S_{B m}(t+1)-S_{B m}(t)+\Delta t{p_{B_{m}}}^{-}(t) \leq\left(1-y 1_{B_{m}}\right) M$

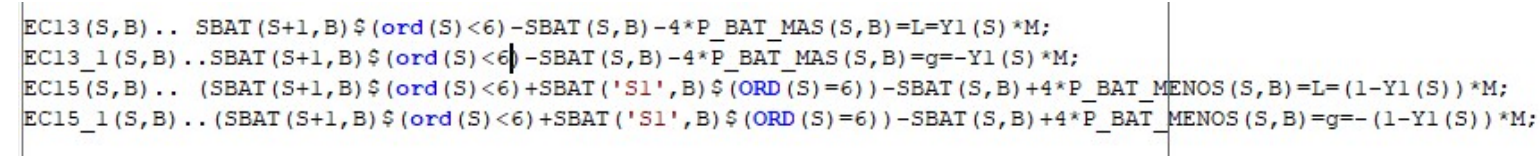




\section{Figure 7}

$0 \leq \delta_{s r}(l) \leq \Delta \delta_{s r}$

A value is assigned for the maximum variation of the partitions of the angle difference between the bars.

$\delta_{s r}^{-} \geq 0$

$\delta_{s r}^{+} \geq 0$

$\delta_{s r} \geq 0$

In Figure 8 the positive variables of the model are defined.

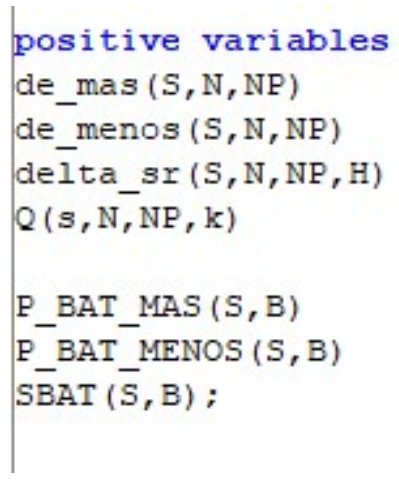

Figure 8

Figure 9 shows the binary variables that, added to the continuous variables, make up the mixed integer programming model.

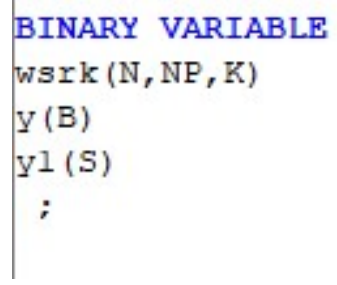

\section{Figure 9}

For the unmodified Garver test system (Figure 10), bids and bids are established by an optimization algorithm supported by market structure. It is assumed that the system load curve is the same for each of the days of the year, likewise for each day 6 scenarios are established in which the demand is constant. The demand coefficient for each of the 6 intervals is summarized in Table 1 . 
Table 1 Demand coefficient

\begin{tabular}{|c|c|}
\hline Scenario & Scenario Demand coefficient \\
\hline 1 & 1 \\
\hline 2 & 1.67 \\
\hline 3 & 3.33 \\
\hline 4 & 2.33 \\
\hline 5 & 2.67 \\
\hline 6 & 2 \\
\hline
\end{tabular}

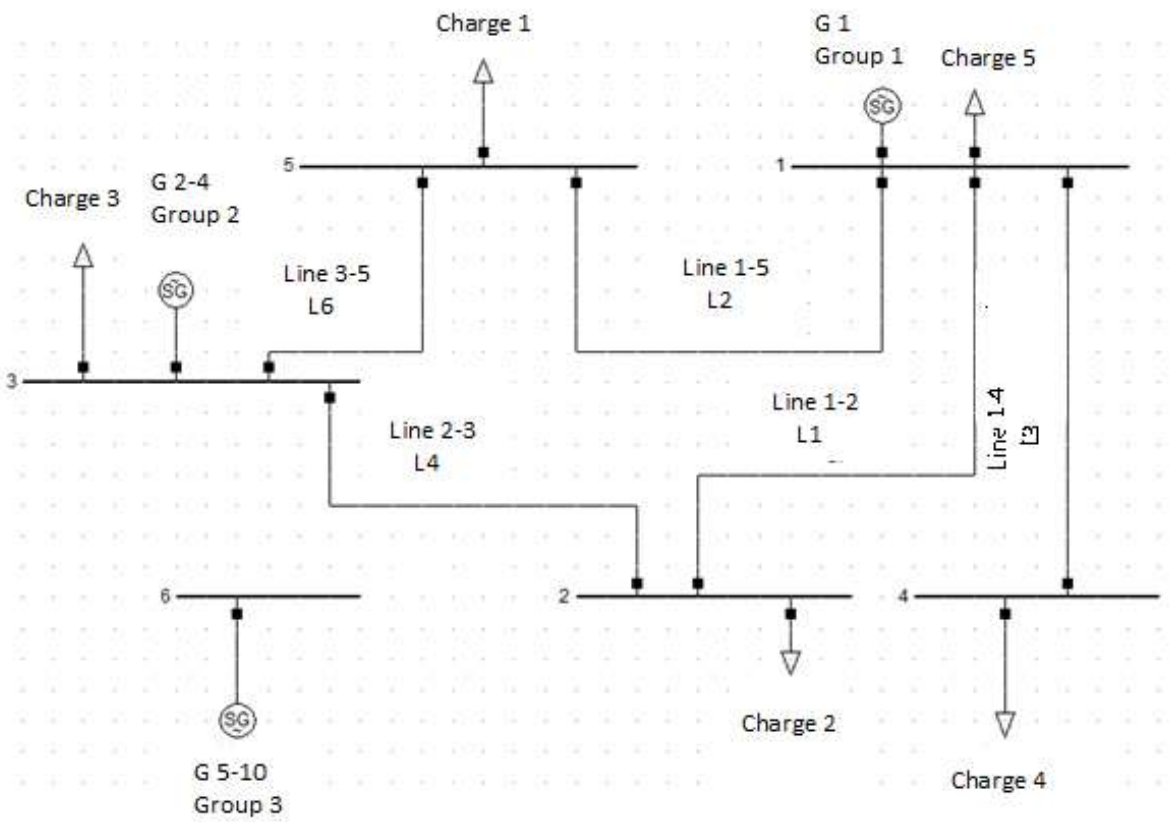

Figure 10: Unmodified Garver system. (Own elaboration)

\section{Loads and generators.}

The maximum power that the generators can deliver and the maximum demand of the loads are described in Table 2 . Driven by a market in perfect competition, the generators establish the sale price per MWh, the D1-D5 demands are divided in turn in 5 equal blocks. Each of the demand blocks has a demand price, which they are willing to pay for each MWh consumed.

Table 2: Power restrictions - bid and ask prices

\begin{tabular}{|c|c|c|c|c|c|c|}
\hline Node & Name & P (MW) & $\begin{array}{c}\text { Offer price (\$ / } \\
\text { MWh) }\end{array}$ & Name & $\begin{array}{c}\text { Power } \\
\text { (MW) }\end{array}$ & Ask Price (\$/MWh) \\
\hline 1 & G1 & 150 & 10 & D1 & 40 & $30,28,26,24,20$ \\
\hline 2 & & & & D2 & 120 & $34,32,30,28,25$ \\
\hline \multirow{2}{*}{3} & G2 & 120 & 20 & D3 & 20 & $20,16,14,12,10$ \\
\cline { 2 - 4 } & G3 & 120 & 22 & & \\
\cline { 2 - 4 } & G4 & 120 & 25 & & & \\
\hline
\end{tabular}




\begin{tabular}{|c|c|c|c|c|c|c|}
\hline 4 & & & & D4 & 80 & $30,27,24,21,17$ \\
\hline 5 & & & & D5 & 120 & $34,30,26,24,18$ \\
\hline \multirow[t]{6}{*}{6} & G5 & 100 & 8 & & & \\
\hline & G6 & 100 & 12 & & & \\
\hline & G7 & 100 & 15 & & & \\
\hline & G8 & 100 & 17 & & & \\
\hline & G9 & 100 & 19 & & & \\
\hline & G10 & 100 & 21 & & & \\
\hline
\end{tabular}

Lines.

Table 3 shows the lines and parameters of the lines of the unmodified Garver 6 node system in figure 1, in addition to the lines that may be built in a possible expansion for the system.

The maximum power expressed in Table 3 refers to the thermal capacity of the line, with a base of 100 MW.

Table 3: Parameters of the system lines

\begin{tabular}{|c|c|c|c|c|c|c|}
\hline Line & $\boldsymbol{R L}(\boldsymbol{p u})$ & $\boldsymbol{X L}(\boldsymbol{p u})$ & $\boldsymbol{b L}(\boldsymbol{p u})$ & $\boldsymbol{g L}(\boldsymbol{p u})$ & $\boldsymbol{P m a x}(\boldsymbol{p u})$ & Cost (M\$) \\
\hline $1-2$ & 0,1 & 0,4 & $-2,353$ & 0,588 & 1 & 40 \\
\hline $1-3$ & 0,09 & 0,38 & $-2,492$ & 0,590 & 1 & 38 \\
\hline $1-4$ & 0,15 & 0,6 & $-1,569$ & 0,392 & 0,8 & 60 \\
\hline $1-5$ & 0,05 & 0,2 & $-4,706$ & 1,176 & 1 & 20 \\
\hline $1-6$ & 0,17 & 0,68 & $-1,384$ & 0,346 & 0,7 & 68 \\
\hline $2-3$ & 0,05 & 0,2 & $-4,706$ & 1,176 & 1 & 20 \\
\hline $2-4$ & 0,1 & 0,4 & $-2,353$ & 0,588 & 1 & 40 \\
\hline $2-5$ & 0,08 & 0,31 & $-3,024$ & 0,780 & 1 & 31 \\
\hline $2-6$ & 0,08 & 0,3 & $-3,112$ & 0,830 & 1 & 30 \\
\hline $3-4$ & 0,15 & 0,59 & $-1,592$ & 0,405 & 0,82 & 59 \\
\hline $3-5$ & 0,05 & 0,2 & $-4,706$ & 1,176 & 1 & 20 \\
\hline $3-6$ & 0,12 & 0,48 & $-1,961$ & 0,490 & 1 & 48 \\
\hline $4-5$ & 0,16 & 0,63 & $-1,491$ & 0,379 & 0,75 & 63 \\
\hline $4-6$ & 0,08 & 0,3 & $-3,112$ & 0,830 & 1 & 30 \\
\hline $5-6$ & 0,15 & 0,61 & $-1,546$ & 0,380 & 0,78 & 61 \\
\hline
\end{tabular}

\section{RESULTS}

Table 4 presents the result obtained by the GAMS model for the values in pu of the power transmitted through the line including losses and with the defined direction from node $s$ to $r$, when no expansion has been performed in the system.

Table 4: GAMS model power flows

\begin{tabular}{|c|c|c|c|c|c|c|}
\hline & \multicolumn{7}{|c|}{ Power Flow (pu) } \\
\hline Line & Scenario 1 & Scenario 2 & Scenario 3 & Scenario 4 & Scenario 5 & Scenario 6 \\
\hline $1_{-} 2$ & 0,413 & 0,36 & 0,336 & 0,296 & 0,335 & 0,31 \\
\hline
\end{tabular}




\begin{tabular}{|c|c|c|c|c|c|c|}
\hline $1 \_4$ & 0,299 & 0,255 & 0,135 & 0,272 & 0,218 & 0,257 \\
\hline $1 \_5$ & 0,467 & 0,484 & 0,672 & 0,558 & 0,52 & 0,561 \\
\hline $2 \_3$ & $-0,878$ & -1 & -1 & -1 & -1 & -1 \\
\hline $2 \_4$ & 0,034 & 0,022 & $-0,13$ & 0,109 & $-0,008$ & 0,074 \\
\hline $3 \_5$ & 0,518 & 0,76 & 1 & 0,965 & 0,847 & 0,94 \\
\hline
\end{tabular}

Table 5 shows the results obtained for the power flow in [1] solved with the GAMS model proposed by the authors.

Table 5: Powers Flow [1]

\begin{tabular}{|c|c|c|c|c|c|c|}
\hline & \multicolumn{7}{|c|}{ Power Flow (pu) } \\
\hline Line & Scenario 1 & Scenario 2 & Scenario 3 & Scenario 4 & Scenario 5 & Scenario 6 \\
\hline $1 \_2$ & 0,4132 & 0,3591 & 0,3402 & 0,3060 & 0,3305 & 0,313 \\
\hline $1 \_4$ & 0,3 & 0,2553 & 0,1375 & 0,2087 & 0,2087 & 0,2087 \\
\hline $1 \_5$ & 0,4665 & 0,4856 & 0,6803 & 0,612 & 0,5341 & 0,5679 \\
\hline $2 \_3$ & $-0,887$ & -1 & -1 & -1 & -1 & -1 \\
\hline $2 \_4$ & 0,0361 & 0,00232 & $-0,133$ & 0,07 & $-0,0169$ & 0 \\
\hline 3_5 & 0,5254 & 0,763 & 1 & 1 & 0,8702 & 0,9399 \\
\hline
\end{tabular}

Table 6 shows the absolute error between the results obtained by the model developed in GAMS by the authors and the results [1]

Table 6: Absolute error between the flows in table 4 and 5

\begin{tabular}{|c|c|c|c|c|c|c|}
\hline & \multicolumn{7}{|c|}{ Absolute relative error (pu) } \\
\hline Line & Scenario 1 & Scenario 2 & Scenario 3 & Scenario 4 & Scenario 5 & Scenario 6 \\
\hline 1_2 & 0,0002 & 0,0009 & 0,0042 & 0,01 & 0,0045 & 0,003 \\
\hline 1_4 & 0,001 & 0,0003 & 0,0025 & 0,0633 & 0,0093 & 0,0483 \\
\hline 1_5 & 0,0005 & 0,0016 & 0,0083 & 0,054 & 0,0141 & 0,0069 \\
\hline 2_3 & 0,009 & 0 & 0 & 0 & 0 & 0 \\
\hline 2_4 & 0,0021 & 0,01968 & 0,003 & 0,039 & 0,0089 & 0,074 \\
\hline 3_5 & 0,0074 & 0,003 & 0 & 0,035 & 0,0232 & $1 \mathrm{E}-04$ \\
\hline
\end{tabular}

The theoretical, practical value and the absolute error of the total net profit are shown in Table 7.

Table 1: Results

\begin{tabular}{|l|l|l|l|}
\hline & Theoretical & Practical & absolute error \\
\hline Total net profit (M\$) & 40,15 & 40,47 & 0,29 \\
\hline
\end{tabular}

\section{CONCLUSIONS}

The implemented mixed integer linear programming model allows evaluating the economic and technical feasibility of incorporating energy storage systems. This mathematical model implemented in GAMS provides improvements from a 
total net benefit point of view by reducing power losses and lowering investment costs in the long term.

\section{REFERENCES}

1. J. A. Aguado, S. de la Torre, and A. Triviño, "Battery energy storage systems in transmission network expansion planning," Electr. Power Syst. Res., vol. 145, pp. 63-72, 2017, doi: 10.1016/j.epsr.2016.11.012.

2. N. Alguacil, A. L. Motto, and A. J. Conejo, "Transmission expansion planning: A mixed-integer LP approach," IEEE Trans. Power Syst., vol. 18, no. 3, pp. 1070-1077, 2003, doi: 10.1109/TPWRS.2003.814891.

3. V. H. Reséndiz Estrada, "Métodos deterministas para planear la expansión de sistemas de transmisión," Inst. Politécnico Nac., p. 250, 2017.

4. S. De Torre, A. J. Conejo, J. Contreras, and S. Member, “in Electricity Markets,” vol. 23, \# 1, pp. 238-248, 2008. 

